\title{
The current status of prophylactic femoral intramedullary nailing for metastatic cancer
}

\author{
NM Ormsby ${ }^{1}$, WY Leong ${ }^{1}$, W Wong ${ }^{2}$, HE Hughes ${ }^{3}$ and V Swaminathan ${ }^{2}$ \\ ${ }^{1}$ Orthopaedic Surgery, Wirral University Teaching Hospital NHS FT, Arrowe Park Road, Upton, Wirral CH49 5PE, UK \\ University of Liverpool, L3 5TR, UK \\ ${ }^{3}$ Palliative Care, Health Education East Midlands, NG11 6NJ, UK
}

Correspondence to: V Swaminathan. E-mail: vikramswaminathan@hotmail.com

\begin{abstract}
The most common site for cancer to spread is bone. At post-mortem, bony metastases have been found in $70 \%$ of patients dying from breast and prostate cancer. Due to the prevalence of cancer, bone metastasis and the associated management represents a huge burden on NHS resources.

In patients with metastasis, around $56 \%$ of these involve the lower limb long bones. Due to the huge forces placed upon long bones during weight bearing, there is a high risk of fracture through areas of metastasis. It is reported that $23 \%$ of pathological fractures occur in the femoral subtrochanteric region. This area is subjected to forces up to four times the body weight, resulting in poor union rate for these fractures, and significant morbidity associated with difficulty in mobilising, and in patient nursing.

As cancer treatments improve, the life expectancy in this subgroup of patients is likely to increase. Therefore medium-to-long-term management of these fractures, beyond the palliative, will become essential. We aim to evaluate the current management for metastatic malignant femoral disease, with particular focus on the prophylactic augmentation of diseased femorii using intramedullary nails.
\end{abstract}

Keywords: metastatic cancer, intramedullary nailing, arthroplasty, internal fixation, pathological fracture, prophylactic nailing

Published: 01/12/2016

Received: 01/06/2016

ecancer 2016, 10:698 DOI: 10.3332/ecancer.2016.698

Copyright: (c) the authors; licensee ecancermedicalscience. This is an Open Access article distributed under the terms of the Creative Commons Attribution License (http://creativecommons.org/licenses/by/3.0), which permits unrestricted use, distribution, and reproduction in any medium, provided the original work is properly cited. 


\section{Introduction}

Cancer currently accounts for one in every four deaths in the United Kingdom [1]. More than one-third of cancers are diagnosed in the over 1975s, and due to an ageing population this statistic is likely to increase [2]. The most common site for cancer spread is bone [3]. The most common cancers to metastasise to bone are breast and prostate. At post-mortem, bony metastases have been found in $70 \%$ of patients dying from breast and prostate cancer [4]. Other malignancies, such as thyroid, kidney, and bronchus, can also spread to bone, with a prevalence of around $30-40 \%$ in those who die from the primary malignancy [4].

Currently, metastasis to bone is associated with an adverse prognosis for these patients, increasing mortality [3]. Significant increased morbidity in these patients causes an increased burden on the health service. In patients with metastasis, around $56 \%$ of these involve the lower limb long bones [3]. Due to the huge forces placed upon long bones during weight bearing, there is a high risk of fracture through areas of metastasis. It is reported that $23 \%$ of pathological fractures occur in the femoral subtrochanteric region [5]. This area is subjected to forces of up to four times of body weight, resulting in poor union rate for these fractures, and significant morbidity associated with difficulty in mobilising, and in patient nursing. Symptoms commonly related to femoral metastatic disease include pain, difficulty weight bearing, and presence of a pathological fracture [6]. Pain from femoral metastasis is associated with subsequent mechanical instability that ultimately results in pathological fracture as the destruction of the bony cortex progresses.

As cancer treatments improve, the life expectancy in this subgroup of patients is likely to increase. Therefore, medium-to-long-term management of these fractures, beyond the palliative, will become essential. There are various surgical interventions that can be considered depending on the site and position of metastatic tumour and whether there is a fracture in situ or an impending fracture. The primary aim is to stabilise the femur, in order to maintain load-bearing ability and prevent subsequent fracture of the weakened cortices secondary to tumour infiltration. Instrumentation of the femur is reported to provide longitudinal structural support and pain relief, allowing the patient to weight bear through the limb $[7,8]$.

These factors are important as they relate to a patient's independence and ability to undertake normal daily activities. Given that some of these patients undergo prophylactic nailing as a palliative procedure, any improvement to quality of life can significantly affect their quality adjusted life years (QALYs). This principle is very important. The prophylactic management of bone metastasis may become an important feature of cancer management in the future. We aim to evaluate the current management for metastatic malignant femoral disease, with particular focus on the prophylactic augmentation of diseased femorii using intramedullary nails.

\section{Diagnosis}

If a malignant lesion is strongly suspected in the femur, further investigations are essential to help confirm their presence, and identify the primary source. Grimer et al. recently published guidance in 2010 relating to the investigations that should be performed when a suspicious bony lesion is suspected [9].

In patients who have a pre-existing diagnosis of cancer, an assumption of metastatic disease must not be made without evidence to support this, as the management plan is vastly different. Bickels et al. have recommended a bone scan to exclude other lesions. If there are none found, then this should be managed like a primary tumour with biopsy to identify a diagnosis [7]. Any other painful sites should also be imaged with plain radiographs.

In patients with no previous history of cancer, a full systematic history and examination is crucial, combined with investigations. Depending on age, two different management pathways are suggested. Recommended imaging modalities include plain radiograph, magnetic resonance imaging $(\mathrm{MRI})$, and isotope bone scan. Bone scans are required as above to identify if this is a solitary lesion or if there is extensive disease. Computed tomography (CT) scans are also crucial to help diagnose primary origin and stage of the disease. They can also be useful to quantify bony destruction and in surgical planning. A tissue diagnosis, however, is key in these patients, guiding the ultimate management of the patient, both from an oncological and from a orthopaedic point of view. If a primary lesion is identified, which is more amenable to biopsy, this is preferable, particularly if the bone is not amenable to surgery or surgery is not currently indicated. 


\section{Biopsy of suspicious lesion}

It is common practice in orthopaedics to send histology samples intraoperatively for patients with pathological lesions and fractures. In patients having arthroplasty surgery or modulated proximal femoral replacement, the excised portion of the femur is sent for suitable tissue analysis by a histopathologist. In those patients with more distal lesions who are being managed with intramedullary device, bone reamings are routinely sent to achieve a tissue diagnosis. A paper by Hassan found that tissue sent from reamings was inadequate in one third of patients, and a diagnosis from reamings alone was not possible for this group of patients [10]. This is due to the traumatic way the tissue is harvested, with significant heat generated by reaming. We should therefore not rely upon this for diagnosis. If at all possible a tissue diagnosis (from a core needle biopsy) would be beneficial prior to any intervention, surgical or otherwise. This enables an MDT decision to be made regarding the multiple important factors that will dictate treatment.

\section{Decision to treat with surgery}

Several different tumour characteristics can influence the way a metastatic lesion is managed and must be considered prior to palliative operative management. It is vital to identify those tumours that respond predictably to oncological therapy without surgical intervention (such as lymphoma), and also primary musculoskeletal tumours (e.g. Sarcoma) that will require urgent specialist referral and intervention [7].

After ruling out the above, the next point is to determine the primary origin of the cancer. This will provide some insight into how aggressive the tumour will be, and how it will affect the bones. Will it cause lytic lesions or sclerotic? It can also be useful in the multi-disciplinary team (MDT) approach as some malignancies will respond better to chemo/radiotherapy and hormonal therapy than others. The origin of the tumour, if known, can also help with pre-operative optimisation. Some malignancies are known to be very vascular, such as bony metastases from a renal cell carcinoma (RCC). Chatziioannou et al. published literature on the significance of embolisation of RCC bone metastases prior to surgery. It was identified that there is a significant difference with blood loss during surgery $(p=0.049)$ and the need for subsequent blood transfusions $(p=0.03)$ when RCC metastases are completely devascularised compared to incomplete devascularisation [11]. These patients may require pre-operative devascularisation in the form of embolisations.

A patient's estimated life expectancy can influence the decision to treat operatively. This is always difficult to assess and it is very individual to each patient. General consensus is that a survival of 6-12 weeks would be the minimum life expectancy required for relatively simple procedures to stabilise a bone such as IM nailing, and 6 months for complex reconstruction procedures [7]. Life expectancy varies greatly between tumour types. Other factors include, premorbid function, local disease control, and degree of spread. There have been many papers looking into positive and negative prognostic indicators in patients with bony metastasis. Pathological fracture, visceral metastasis, low haemoglobin ( $<7 \mathrm{mmol} / \mathrm{L}$ ) and lung cancer were found to be negative predictors in one study, with myeloma positively prognostic [12]. A study by Nathan et al. found that independent negative predictors were performance status, number of bone metastases, visceral metastases, haemoglobin count. Interestingly, the most stastically significant prognostic value was the surgeon's survival estimate itself [13].

\section{Isolated solitary lesion}

Identification of patients with an isolated solitary metastasis warrants further more detailed assessment. The desire is often to fully excise localised solitary lesions, however, given that the disease is systemic by definition this is not curative. There is evidence showing that radical excision of solitary metastasis can provide a more prolonged disease-free interval and thus improving patient's survivability [14]. This is particularly true for metastasis from renal cell carcinoma [15]. Baloch et al. reported in his case series of 25 patients one, three and five year cumulative survival rates of $88 \%, 54 \%$, and $13 \%$, respectively, following either, radical excision, amputations or with endoprosthetic replacement [14]. Hence, if patients demonstrated higher survivability outcome, resection with reconstruction may provide much more durable and reliable construct. Conversely, here is evidence that resection of lesions does not improve patient outcomes, but in general, patients with solitary lesions do better than those with multiple bony deposits [7]. 


\section{Pathological fractures}

An untreated bone metastasis can lead to a pathological fracture. This is one of the most important factors affecting patient morbidity. The mechanism of how a tumour causes bony destruction is important to understand. There are three distinct phenotypes of bony metastasis: bony lysis, sclerotic lesions, and a mixed picture. This impacts the risk of pathological fracture. Osteolytic lesions (the classic being breast) stimulate osteoclastogenesis through tumour production of interleukin (IL)-1, IL-6, MIP1a, and RANK ligand. The tumour also stimulates existing osteoclasts through the production of tumour necrosis factor (TNF) and parathyroid hormone-related peptide, increasing RANK-L expression on osteoblasts [16]. Osteoblastic lesions (classically prostate) are the result of tumour cell-mediated activation of osteoblasts, through production of TGF- $\beta$ and bone morphogenic protein [17]. Prostate tumour cells also demonstrate osteomimicry with the tumour cell differentiating towards an osteoblastic bone forming phenotype [18, 19]. Fractures will commonly occur through the area of metastatic lesions, especially in weight bearing bones. Features suggestive of impending pathological fracture include large lesions, lytic in nature, and erosion into or through a cortex of the femoral bone.

\section{Estimating the risk of pathological fracture}

Once a metastatic lesion has been identified in the femur, tools are available to help quantify the risk of a pathological fracture. A scoring system for predicting this risk was originally developed by Harrington in 1986. He listed four criteria that could be used to predict the fracture risk of metastatic disease primarily in the femur (Figure 1). Previous studies state that the existence of one or more of the described criteria is thought to be an indication for prophylactic fixation of femur. However, limited statistical evidence is available to describe the sensitivity, specificity and reproducibility of these criteria [20-22].

Mirels developed another scoring system in 1989, which is currently widely used to predict the risk of pathological fracture. This scoring system consists of four criteria, each scored from 1 to 3 (Figure 2). A score of $\leq 7$ and $\geq 9$ gives a fracture risk of $4 \%$ and $33 \%$, respectively. He further recommended radiotherapy for patients with a score of $\leq 7$, clinical judgement for a score of 8 and prophylactic fixation for patients with a score of $\geq 9$. Several studies have validated Mirel's scoring system, showing sensitivity and specificity to be $91 \%$ and $35 \%$, respectively [23, 24 ].

There are limitations with the Mirels' scoring; the low specificity and uncertainty with a score of 8 could lead to potentially unnecessary interventions being performed in cancer patients.

Recently, CT scan has been developed as an assessment tool for risk of impending fracture. Nazarian et al had developed and validated CT-based rigidity analysis (CTRA). CT scans of the bone involved and a hydroxyapatite phantom (CIRS Tissue Simulation and Phantom Technology) to standardise X-ray attenuation for each pixel to bone mineral density. The final calculation provides a measure of bone resistance to Uniaxial loads (EA), bending moment (EI) and torsional moment (GJ) (Figure 3).

The CTRA was found to have a sensitivity of $100 \%$ versus $67 \%$ and specificity of $60 \%$ versus $48 \%$ when comparing to Mirel scores (Mirel score $\geq 9$ ). Folowing initial consultation, patients who were deemed to have high risk of impending fractures received stabilisation and were excluded from Damron et al. study. Hence, the study can only be generalised to patient population whereby the benefit of prophylactic stabilisation was questionable following initial assessment.

Despite the clear superiority in sensitivity and specificity, it is worth pointing out that CTRA is not as easily accessible in a clinical setting. In some cases, it will be quite apparent from clinical assessment and Mirel scoring regarding the impending risk of fracture. In cases whereby Mirel scoring were inconclusive, CTRA can be performed to provide further information which might guide treatment [25-26].

\begin{tabular}{|l|}
\hline Harrington's criteria for predicting risk of fracture. \\
\hline Cortical bone destruction $>50 \%$ \\
\hline A lesion $>2.5 \mathrm{~cm}$ in the proximal femur \\
\hline Destruction of subtrochanteric femoral region \\
\hline Persistent pain despite irradiation \\
\hline
\end{tabular}

Figure 1. Harrington's criteria for predicting risk of pathological fracture. 


\begin{tabular}{|l|l|l|l|}
\hline \multicolumn{4}{|l|}{ Mirels' scoring system for pathological fracture prediction. } \\
\hline Score & 1 & 2 & 3 \\
\hline Site & Upper limb & Lower limb & Trochanteric \\
\hline Pain & Mild & Moderate & Functional \\
\hline X-ray appearance & Blastic & Mixed & Lytic \\
\hline Size of lesion & $<1 / 3$ Cortex & $1 / 3-2 / 3$ cortex & $>2 / 3$ cortex \\
\hline
\end{tabular}

Figure 2. Mirels' scoring system for pathological fracture prediction.

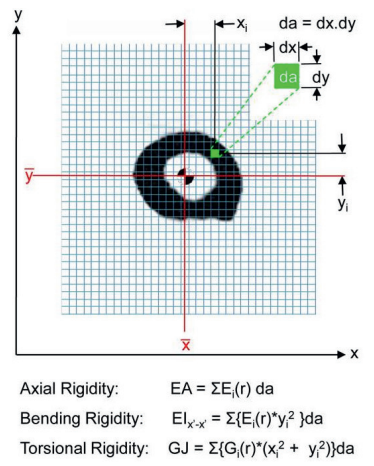

Figure 3. CT-based rigidity analysis (CTRA).

Tatar et al. also examined the correlation of size of long bone metastasis with percentage of cortical bone involvement measured on CT scan to risk of fractures in 47 patients who were treated with radiotherapy. They have concluded that the only positive predictive parameter for fracture was circumferential cortical involvement of $\geq 30 \%$ with recommendation of nailing for any patients within this group in their Level IV study [27].

\section{Indications for surgical management}

The major indications for surgical management of femoral metastasis are in patients with impending or pathological fractures, and in those with intractable pain [28-30]. Several options are available for surgical management. Prophylactic internal fixation of the femur for metastatic disease was first described by Griessman et al in 1947. Since then, a number of case reports and studies have been published with intramedullary nailing becoming the treatment of choice for impending femoral fractures caused by metastases [31]. A recent study showed that patients who underwent prophylactic fixation have improved post-operative outcomes than patients who underwent fixation after pathological fracture, including longer survival, and shorter hospital stay [32].

\section{Nailing}

Despite a long history of performing intramedullary nailing, limited evidence exists which evaluates the most appropriate surgical implantation technique. Historically, cephalomedullary nails were used for prophylactic fixation as it provides greater stability for the entire femur including the femoral neck as opposed to plating. However, conflicting evidence has been identified in different studies and it remains uncertain if femoral neck fixation is necessary in patients with subtrochanteric metastatic lesions. Study by Moon et al found no femoral neck lesions in follow-up of 145 nailings in 141 patients with subtrochanteric metastatic lesion. Hence, they do not recommend stabilising the femoral neck in these group of patients [33]. Alvi and Damron found incidence of disease progression was considerably lower than the complication rate potentially attributable to the use of cephalomedullary implants, but this is very difficult to say categorically. Only one patient in this series developed a new lesion not previously recognised, but $12 \%$ progressed [34]. Fixation with nails is better than plates 
and screws, although not periprosthetic [32]. Biomechanically speaking a reamed nail does provide a more stable construct in comparison to undreamed nail. However, Cole et al., in their paper suggested that there is evidence that reaming is no better, and that reamings are not ideal diagnostically. Hence, undreamed nails would be best, reducing trauma and operative time [35].

Venting is a method developed to reduce the intramedullary pressure during nail insertion. It has been hypothesised that a raised intramedullary pressure could lead to some surgical complications. A study showed that venting could reduce intramedullary pressure in a femur with metastatic disease, but not low enough to prevent fat and tumour embolisations. It is also unclear how venting affects the spreading of cancer locally and systematically [36]. A questionnaire has been developed for orthopaedic surgeons in Canada which asked if they routinely use venting when treating impending femoral fractures. The results showed that there is no general consensus regarding the routine use of venting [37].

\section{Complications of intramedullary nailing}

Several complications are associated with prophylactic fixation of metastatic femoral disease. These include general surgical risks such as infection (1-2\%), deep vein thrombosis $(4.9-14 \%)$, and intraoperative (1\%) or postoperative death (3 months mortality rate of roughly $13 \%$, 1-year mortality rate of $70-90 \%$ depending on cancer type) with mean post-operative survival time of 14 months [32, 38, 39]. Procedurespecific risks include fat embolisation, failure of implant (2-8\%), seeding of cancer, and periprosthetic fracture. Overall, complication rate related to surgery is approximately $8 \%[32,38,39]$. There remains uncertainty with regard to the effects of intramedullary nailing on local and systematic spreading of cancer with some studies indicating that there are no significant effects, while others identified some evidence of the tumour spreading [31].

\section{Arthroplasty}

As the proximal femur is one of the commonest sites of bone metastases, arthroplasty has a role in managing impending proximal femur fractures. Arthroplasties are often performed when there is acetabular involvement or presence of severe bony destruction and when any attempts of internal fixation are likely to be frutile. Surgical resection of the tumours followed by endoprosthetic reconstruction is a proven treatment option and allows for immediate weight bearing and reducing risks of construct failure post-surgery. However, these procedures are very complicated and should only be performed in specialist orthopaedic centres [38, 40-42].

\section{Plating}

When the distal femur is affected, plating could be used as an option to stabilise the affected part of the femur. Generally, intramedullary nails are the devices of choice for fixation as they offer more extensive stabilisation of bone, a reduced risk of future fracture and a lower rate of fixation failure as opposed to plating. We were, however, unable to find any articles comparing the prophylatic use of intramedullary nailing and plating. Plating is generally reserved for pathological fractures where the use of intramedullary devices are contraindicated, such as the presence of densely sclerotic lesions or when an unstable metaphyseal fragments that could not be stabilised with intramedullary construct are present [43].

\section{Non-operative treatment}

Metastatic bone disease is complicated and treatment requires a MDT approach to provide patients with the best outcome. Depending on the size of tumour and the patient's comorbidities, metastatic femoral disease could primarily or initially be managed medically. Treatment options include chemotherapy, radiotherapy, bisphosphonates and hormonal therapy. Bisphosphonates have been found to reduce bone pain and delay the development of complications to bone [44]. No coherent data between studies exists on fracture risks following medical management of metastatic femoral disease; some papers suggested roughly $5-10 \%$ risk of fracture [32, 38]. Others show no difference between surgically or medically treated patients and some favour surgical management [45]. It is however noted that all these papers have a relatively small sample size and results published may have low statistical power. 


\section{Fitness for surgery}

Regardless of surgical techniques available, a patient must ultimately be a candidate for surgery. Patient selection is always an important influencing factor, and is more complicated particularly in patients with metastatic disease. The assessment for suitability for surgical measures also becomes increasingly important in the elderly population, given their associated health confounding comorbidities; age alone however is not an accurate independent predictor of surgical outcome [46]. Often it is these comorbidities or perceived fitness status that declares a patient unsuitable for surgical management [47]. The concept of frailty further confounds the decision to proceed to surgery, especially in an older patient. A patient's current status with regards to daily activities, mobility and functioning are all highly relevant to prophylactic surgery of the femur. An evaluation needs to be made to ascertain the potential benefit gained from surgery. Previous functional status prior to metastasis to the affected limb, alongside prognostic values for the primary disease are required information. A multidisciplinary approach is therefore a must for these patients.

\section{Conclusions}

Changing demographics and an ageing population forecast an increased incidence in cancer and consequential secondary complications such as bone metastases. There is therefore an essential growing need to establish which management pathway and method of prophylaxis for pathological fracture is the most effective in improving prognosis. Inevitably, establishing a universal solution is difficult solely due to the complexity of cancer treatment. Treatment is dependent on each particular patient and their bespoke health needs and fitness.

With criteria available to help recognise patients at high risk from femoral shaft fracture, it is important to have practical preventative interventions in place. These treatments will undoubtedly become increasingly relevant in the future of orthopaedics. Prophylactic fixation aims to stabilise the affected area and therefore prevent pathological fractures, in addition to improving mobility, patient prognosis and quality of life. There is reason to suggest that intramedullary nails could be used for effective prophylaxis, however the current lack of evidence surrounding surgical interventions is not sufficient to concretely justify which method has the most potential. The authors propose to create a pragmatic algorithm for the diagnosing clinican for the management of these fractures, and plan to report these findings upon completion.

\section{References}

1. UK Cr Cancer mortality statistics 2011 [Available from: http://www.cancerresearchuk.org/health-professional/cancer-mortality-statistics

2. Gavrilov LA, Heuveline P (2003) Aging of population (Eds.) PDaGM, editor: New York, Macmillan Reference USA

3. Coleman RE (2006) Clinical features of metastatic bone disease and risk of skeletal morbidity Clin Cancer Res 12(20 Pt2) 6243s-9s DOI: 10.1158/1078-0432.CCR-06-0931 PMID: 17062708

4. Galasko C (1981) The anatomy and pathways of skeletal metastases Boston: GK Hall

5. Weikert DR, Schwartz HS (1991) Intramedullary nailing for impending pathological subtrochanteric fractures $J$ Bone Joint Surg Br 73(4) 668-70 PMID: 2071657

6. Mercadante S (1997) Malignant bone pain: pathophysiology and treatment Pain 69(1-2) 1-18 DOI: 10.1016/S03043959(96)03267-8 PMID: 9060007

7. Bickels J, Dadia S and Lidar Z (2009) Surgical management of metastatic bone disease J Bone Joint Surg Am 91(6) 1503-16 DOI: 10.2106/JBJS.H.00175 PMID: 19487532

8. van der Hulst RR, van den Wildenberg FA and Vroemen JP et al. (1994) Intramedullary nailing of (impending) pathologic fractures J Trauma 36(2) 211-5 DOI: 10.1097/00005373-199402000-00012 PMID: $\underline{8114139}$

9. Grimer R, Mottard S and Briggs T (2010) Focus on earlier diagnosis of bone and soft-tissue tumours Brit Ed JBJS

10. Hassan K, Kalra S and Moran C (2007) Intramedullary reamings for the histological diagnosis of suspected pathological fractures Surgeon 5(4) 202-4 DOI: 10.1016/S1479-666X(07)80003-5 PMID: 17849954 
11. Chatziioannou AN, Johnson ME and Pneumaticos SG et al (2000) Preoperative embolization of bone metastases from renal cell carcinoma Eur Radiol 10(4) 593-6 DOI: 10.1007/s003300050969 PMID: 10795540

12. Hansen BH, Keller J and Laitinen M et al (2004) The Scandinavian Sarcoma Group Skeletal Metastasis Register Survival after surgery for bone metastases in the pelvis and extremities Acta Orthop Scand Supp/ 75(311) 11-5 D0I: 10.1080/00016470410001708270 PMID: 15188660

13. Nathan SS, Healey JH and Mellano D et al (2005) Survival in patients operated on for pathologic fracture: implications for endof-life orthopedic care J Clin Oncol 23(25) 6072-82 DOI: 10.1200/JCO.2005.08.104 PMID: 16135474

14. Baloch KG, Grimer RJ and Carter SR et al (2000) Radical surgery for the solitary bony metastasis from renal-cell carcinoma J Bone Joint Surg Br 82(1) 62-7 DOI: 10.1302/0301-620X.82B1.9995 PMID: 10697316

15. Takashi M, Takagi $Y$ and Sakata $T$ et al (1995) Surgical treatment of renal cell carcinoma metastases: prognostic significance Int Urol Nephrol 27(1) 1-8 DOI: 10.1007/BF02575213 PMID: 7615361

16. Roodman GD (2004) Mechanisms of bone metastasis N Engl J Med 350(16) 1655-64 DOI: 10.1056/NEJMra030831 PMID: 15084698

17. Logothetis CJ, Lin SH (2005) Osteoblasts in prostate cancer metastasis to bone Nat Rev Cancer 5(1) 21-8 DOI: 10.1038/nrc1528 PMID: 15630412

18. Koeneman KS, Yeung F and Chung LW (1999) Osteomimetic properties of prostate cancer cells: a hypothesis supporting the predilection of prostate cancer metastasis and growth in the bone environment Prostate 39(4) 246-61 PMID: 10344214

19. Lin DL, Tarnowski CP and Zhang J et al (2001) Bone metastatic LNCaP-derivative C4-2B prostate cancer cell line mineralizes in vitro Prostate 47(3) 212-21 DOI: 10.1002/pros.1065 PMID: 11351351

20. Piccioli A, Spinelli MS and Maccauro G (2014) Impending fracture: a difficult diagnosis Injury 45 S138-41 DOI: 10.1016/j. injury.2014.10.038 PMID: 25457334

21. Harrington KD (1986) Impending pathologic fractures from metastatic malignancy: evaluation and management Instr Course Lect 35 357-81 PMID: $\underline{3819423}$

22. Ashford R (2009) Palliative orthopaedic surgery for skeletal metastases Eur Oncol 5(1) 30-4

23. Mirels $\mathrm{H}$ (1989) Metastatic disease in long bones: A proposed scoring system for diagnosing impending pathologic fractures Clin Orthop Relat Res 2003(415 Suppl) S4-13

24. Jawad MU and Scully SP (2010) In brief: classifications in brief: Mirels' classification: metastatic disease in long bones and impending pathologic fracture Clin Orthop Relat Res 468(10) 2825-7 DOI: 10.1007/s11999-010-1326-4 PMID: 20352387 PMCID: $\underline{3049613}$

25. Nazarian Ara, Entezari $V$ and Zurakowski D et al (2015) Treatment planning and fracture prediction in patients with skeletal metastasis with CT-based rigidity analysis Clin Cancer Res 21(11) 2514-9 DOI: 10.1158/1078-0432.CCR-14-2668 PMID: 25724521 PMCID: $\underline{4452435}$

26. Damron T, Nazarian A and Entezari $V$ et al (2016) CT-based structural rigidity analysis is more accurate than Mirels scoring for fracture prediction in metastatic femoral lesions Clini Orthop Relat Res 474 643-51 DOI: 10.1007/s11999-015-4453-0

27. Tatar Z, Soubier M and Dilles AF et al (2014) Assessment of the risk factors for impending fractures following radiotherapy for long bone metastases using CT scan-based virtual simulation: a retrospective study Radiation Oncol 922 DOI: $10.1186 /$ $\underline{\text { s13014-014-0227-1 }}$

28. Kollender $\mathrm{Y}$, Bickels $\mathrm{J}$ and Price WM et al (2000) Metastatic renal cell carcinoma of bone: indications and technique of surgical intervention J Urol 164(5) 1505-8 DOI: 10.1016/S0022-5347(05)67016-4 PMID: 11025692 
29. Bickels J, Kollender $Y$ and Wittig JC et al (2005) Function after resection of humeral metastases: analysis of 59 consecutive patients Clin Orthop Relat Res 2005(437) 201-8 DOI: 10.1097/01.blo.0000165857.12676.00

30. Harrington KD (1981) The management of acetabular insufficiency secondary to metastatic malignant disease $J$ Bone Joint Surg Am 63(4) 653-64 DOI: 10.2106/00004623-198163040-00017 PMID: 6163784

31. Fidler M (1973) Prophylactic internal fixation of secondary neoplastic deposits in long bones $\mathrm{Br} M e d \mathrm{~J}$ 1(5849) 341-3 DOI: 10.1136/bmj.1.5849.341 PMID: $\underline{4685628}$ PMCID: $\underline{1588223}$

32. Arvinius C, Parra JL and Mateo LS et al (2014) Benefits of early intramedullary nailing in femoral metastases Int Orthop 38(1) 129-32 DOI: $\underline{10.1007 / s 00264-013-2108-x ~ P M C I D: ~} \underline{3890135}$

33. Moon B, Lin P and Satcher $R$ et al (2015) Intramedullary nailing of femoral diaphyseal metastases: is it necessary to protect the femoral neck? Clin Orthop Relat Res 473(4) 1499-502 DOI: 10.1007/s11999-014-4064-1 PMCID: 4353549

34. Alvi HM and Damron TA (2013) Prophylactic stabilization for bone metastases, myeloma, or lymphoma: do we need to protect the entire bone? Clin Orthop Relat Res 471(3) 706-14 DOI: 10.1007/s11999-012-2656-1 PMCID: 3563787

35. Cole AS, Hill GA and Theologis TN et al (2000) Femoral nailing for metastatic disease of the femur: a comparison of reamed and unreamed femoral nailing Injury 31(1) 25-31 DOI: 10.1016/S0020-1383(99)00195-3 PMID: 10716047

36. Roth SE, Rebello MM and Kreder $\mathrm{H}$ et al (2004) Pressurization of the metastatic femur during prophylactic intramedullary nail fixation J Trauma 57(2) 333-9 DOI: 10.1097/01.TA.0000082157.29723.16 PMID: 15345982

37. Dalgorf D, Borkhoff CM and Stephen DJ et al (2003) Venting during prophylactic nailing for femoral metastases: current orthopedic practice Can J Surg 46(6) 427-31 PMID: 14680349 PMCID: $\underline{3211767}$

38. Wedin R and Bauer HC (2005) Surgical treatment of skeletal metastatic lesions of the proximal femur: endoprosthesis or reconstruction nail? J Bone Joint Surg Br 87(12) 1653-7 DOI: 10.1302/0301-620X.87B12.16629 PMID: 16326880

39. Ristevski B, Jenkinson RJ and Stephen DJ et al (2009) Mortality and complications following stabilization of femoral metastatic lesions: a population-based study of regional variation and outcome Can J Surg 52(4) 302-8 PMID: 19680515 PMCID: 2724796

40. Bickels J, Meller I and Henshaw R et al (2001) Proximal and total femur resection with endoprosthetic reconstruction Musculoskelet Cancer Surg 439-58

41. Plancarte R, Guajardo $\mathrm{J}$ and Meneses-Garcia A et al (2014) Clinical benefits of femoroplasty: a nonsurgical alternative for the management of femoral metastases Pain Physician 17(3) 227-34 PMID: 24850104

42. Haidukewych $G$ (2012) Metastatic disease around the hip: maintaining quality of life JBJS Brit 94(11) 22-5

43. Healey JH and Brown HK (2000) Complications of bone metastases: surgical management Cancer 88(12 Suppl) 2940-51 PMID: 10898338

44. Riccio Al, Wodajo FM and Malawer M (2007) Metastatic carcinoma of the long bones Am Fam Physician 76(10) 1489-94 PMID: 18052014

45. Cheng DS, Seitz CB and Eyre HJ (1980) Nonoperative management of femoral, humeral, and acetabular metastases in patients with breast carcinoma Cancer 45(7) 1533-7 PMID: 7370912

46. Maas HA, Janssen-Heijnen ML and Olde Rikkert MG et al (2007) Comprehensive geriatric assessment and its clinical impact in oncology Eur J Cancer 43(15) 2161-9 DOI: 10.1016/j.ejca.2007.08.002 PMID: 17855074

47. Vicini E, Swaminathan V and Audisio R (2014) Management of breast cancer in elderly patients Clin Pract 11(1) 59-69 DOI: $10.2217 / \mathrm{cpr} .13 .73$ 